\title{
Proceso de Bolonia (II): educación centrada en el que aprende
}

\author{
J. Prat-Corominas ${ }^{a, d}$, J. Palés-Argullós ${ }^{a, b}$, M. Nolla-Domenjóa ${ }^{a, c}$, A. Oriol-Bosch ${ }^{a}, A$. Gual ${ }^{a, b}$
}

El proceso de Bolonia apunta hacia la educación centrada en el que aprende. Lo hace desde una perspectiva administrativa cuando instaura el nuevo sistema de medida académica, el ECTS (European Credit Transferable System), común para todos y con la finalidad obvia de gestionar las transferencias de crédito de los programas Erasmus. Esta unidad de medida, a diferencia del crédito académico antiguo, que sólo contabilizaba como carga de trabajo las horas presenciales, es decir, el tiempo de ocupación del profesor, se modifica para incorporar las horas de trabajo del alumno, con y sin la presencia de un docente. Pero el mensaje no ofrece dudas a estas alturas. La universidad en Europa percibe que la sociedad le está pidiendo que sirva a un fin tan práctico como conseguir que el alumno aprenda y que lo considere un adulto responsable de su propio aprendizaje [1]. Como esto es relativamente nuevo en nuestro entorno parece recomendable iniciar un proceso de reflexión sobre lo que representa la educación centrada en el que aprende y cómo puede plantearse la migración desde la educación dirigida por el que enseña hasta esta nueva meta.

La educación que se orienta a la obtención de resultados debe entenderse como un proceso transformacional de los que aprenden. Para la educación médica se trata de la transformación de jóvenes 'legos' en médicos. Los estudiantes son los que aprenden; por tanto, el complejo proceso transformacional del que aprende es lo que debería guiar las estrategias educativas institucionales y las tareas de los docentes.

Sin embargo, las cosas no han discurrido hasta ahora por estos cauces. El diseño curricular, por un lado, no ha conseguido superar los esquemas clásicos de las asignaturas centradas en el corpus de conocimiento del área del profesor, como unidad estructural básica, y en los ciclos, que permiten segregar, con graves consecuencias para el aprendizaje, el razonamiento científico de base de la aplicación clínica. Su implementación y monitorización, por otra parte, se han centrado en las asignaciones docentes y en el control de su realización por los departamentos y profesores responsables. Si los que enseñan cumplen con las exigencias establecidas y los estudiantes los siguen atentamente, los resultados se consideran satisfactorios. Ésta ha sido la creencia del sistema tradicional, centrado en los procesos.

Los avances de las últimas décadas en las ciencias cognitivas, sin embargo, han permitido elaborar un paradigma 'constructivista' del aprendizaje en el que la motivación personal para aprender desempeña un papel esencial. Según este paradigma constructivista, el aprendizaje es un fenómeno singular e individual, que puede verse facilitado por la interacción en grupo, pero que es idiosincrásico, puesto que no se aprende desde tabula rasa, es decir, desde el no saber nada, sino que se aprende a partir de lo que ya se sabe, se construye sobre las experiencias educativas previas.

El paradigma constructivista del aprendizaje requiere saber qué es lo que llamamos 'conocimiento’ y su carácter utilitarista, cómo se organiza y reorganiza permanentemente el archivo mental que denominamos 'memoria' y cómo se movilizan sus contenidos, cómo se construyen las 'rutinas' con sus ventajas y limitaciones y, finalmente, cómo se identifican y definen los problemas y las estrategias para solucionarlos.
Fundación Educación Médica (FEM).

b Facultad de Medicina. Universitat de Barcelona.

c Fundació Doctor Robert. Universitat Autònoma de Barcelona.

d Universitat de Lleida.

Correspondencia Dr. Jordi Palés Argullós. Departamento de Ciencias Fisiológicas I. Facultad de Medicina. Universitat de Barcelona. Casanova, 143 E-08036 Barcelona.

E-mail jpales@ub.edu 
Estas cuestiones, aunque puedan ser generalizables en términos abstractos, en la vida real se producen contextualizados y su análisis se lleva a cabo en situaciones concretas, que, en nuestro caso, lo constituye la actuación profesional del médico. Y si el proceso educativo tiene como finalidad que el que aprende llegue a ser capaz de exhibir una actuación previsible basada en un correcto razonamiento científico y ético, que corresponda a la de un profesional competente, será necesario conocer cuáles son características de la actuación profesional del médico a fin de facilitar, como educadores, la manera más eficaz y eficiente para lograrlo. No debe olvidarse que la educación no es un fin en sí misma y que la educación médica tiene como objetivo primordial que los estudiantes de grado y los recién graduados aprendan a ejercer la profesión médica para mejorar la atención a la salud de los pacientes y a la población en general. Se trata de una formación profesional para ejercer una práctica social que requiere ser ejercida con excelencia. Porque el ejercicio de la medicina, no debe olvidarse, es una practica relacional con los pacientes y familiares, en primer término, y con los colegas y otros profesionales sanitarios en distintos entornos clínicos, así como con la comunidad, en general.

La conceptualización clásica convencional de la educación médica se centra en la adquisición por parte del que aprende, estudiante o residente, de los conocimientos y habilidades de las ciencias básicas y clínicas. Comienza ahora a reconocerse que el ejercicio profesional requiere asimismo tener la capacidad para su integración en las estructuras sociales, asumiendo roles no clínicos, reconocer las funciones de otros agentes sanitarios y saber utilizar los recursos disponibles. Todo ello debe ser tenido en cuenta por los sistemas de educación médica para dar respuesta a las necesidades de la sociedad.

\section{Aprendiendo a ejercer la medicina}

Hoy, como ayer y mañana, la atención a los pacientes es la actividad nuclear del ejercicio de la medicina. Haber desarrollado una mentalidad inquisitiva y el deseo de mejora son dos componentes básicos para ejercer adecuadamente las funciones de la profesión médica. Estar al día de los avances, integrando e incorporando nue- vos conocimientos, constituye una obligación profesional que debe convertirse en una rutina diaria. En gran medida ello se produce interactuando con colegas y otros agentes sanitarios, al tiempo que se mejora e innova el funcionamiento del equipo y del sistema asistencial, puesto que el médico está formando parte de una comunidad profesional capaz de responder a las necesidades del entorno, y gran parte de la eficacia de su labor depende de la calidad de la interacción dentro de esa comunidad. En esta comunidad profesional se despliegan papeles y funciones no clínicas que están en expansión permanente y que deben adquirirse en el proceso formativo no tan sólo como un objetivo estático, sino como una capacidad y una voluntad evolutiva. La atención a los pacientes, la mejora permanente y la participación en la comunidad profesional son tres componentes básicos de la medicina.

Las facultades de medicina y el sistema residencial de formación especializada de posgrado transforman las personas que aprenden en profesionales de la medicina, es decir, forjan una identidad profesional. Esta transformación es tanto un proceso de desarrollo personal (devenir) como social (contribuir), que se ha definido como 'un proceso permanente autorreflexivo de los hábitos de pensar, sentir y actuar' [2]. El médico que necesita la sociedad ha de estar comprometido y sentirse responsable frente a los pacientes, los colegas, las instituciones, la sociedad y él mismo, así como estar imbuido de la aspiración a mejorar su actuación y conseguir siempre mejores resultados.

Aprender a atender a los pacientes es fundamentalmente un proceso experiencial, es decir, la destreza se adquiere en el hacerlo repetidamente. Si la formación recibida se lleva a cabo de forma primordial en las aulas, aprender en los contextos clínicos constituye una novedad que exige preparación, actitud y reflexión sobre aquellas actividades prácticas que va a aprender. Debe además concebir los pacientes holísticamente y entender los contextos y los distintos condicionantes de los resultados clínicos. Por ello, el sistema educativo deberá considerar diversos factores para la formación clínica:

- La adquisición de 'experteza' es un proceso progresivo.

- El conocimiento y la 'experteza' poseen una naturaleza dinámica y situacional.

- Las formas de aprender, saber y razonar son múltiples y variadas. 
- La inteligencia está distribuida en toda la comunidad clínica.

La evolución de la pericia se ha estudiado comparando el contenido del conocimiento de los 'novatos' con el de los expertos [3], las estructuras de su conocimiento [4], los distintos enfoques del razonamiento clínico [5] y el desempeño de habilidades procedimentales [6]. Frente a este enfoque estático, que ignora los medios utilizados para el aprendizaje, otros estudiaron los procesos por los que se deviene un experto [7] o compararon los que devienen expertos con aquellos que después de años de experiencia no lo logran [8]. El enfoque dinámico del desarrollo de la pericia considera tanto el proceso de adquisición de rutinas como otros de mayor complejidad, como la recogida y procesamiento de información, el respeto y la empatía en el trato, la negociación de alternativas terapéuticas con los pacientes y sus familiares, o la evaluación de los resultados y el desarrollo de planes para mejorarlos.

El reconocimiento de la naturaleza dinámica de la competencia es consecuencia del cambio permanente del conocimiento médico, lo que conlleva a modificar la forma de concebir el contenido curricular y los objetivos de aprendizaje en su formación. Desde la perspectiva sociocultural, es necesario tener en consideración, junto a los conocimientos científicos y clínicos exigibles, el compromiso de servicio que impregna la labor profesional.

Los médicos utilizan diversas formas de conocimiento y diferentes estrategias de razonamiento cuando atienden a sus pacientes [9]. A lo largo del continuo educativo, aparecen distintos tipos de conocimiento y de razonamiento, desde las ciencias biomédicas que se centran en los hechos, la comprensión conceptual y el razonamiento causal, a la orientación pragmática de la práctica clínica que utiliza el conocimiento tácito y situacional para dirigir la actuación [10]. Los estudiantes y residentes, a lo largo de su proceso formativo, van utilizando combinaciones distintas de formas de razonamiento analítico y no analítico, según el conocimiento y la experiencia que dispongan en cada situación clínica. Si lo que se observa evoca situaciones ya vividas será posible orientar los pasos siguientes utilizando fácilmente el conocimiento tácito (inconsciente) que se posee, lo que facilita la detección de elementos secundarios y sutiles del caso. Sin embargo, si la situación no es reconocible con la experiencia disponible, será necesario utilizar recursos de conocimiento formal explícito y razonamiento causal de mayor laboriosidad.

La cuestión para los educadores consiste en encontrar la mejor manera de desarrollar estas formas de conocimiento y de razonamiento que les permite pasar al razonamiento causal o analítico cuando se hallan frente a problemas para los que los procesos rutinarios no son suficientes, evitando así la comisión de errores [11]. La visión tradicional favorece la adquisición del conocimiento científico de los hechos como primera fase del aprendizaje: currículo dividido en dos ciclos, el básico y el clínico, con contenidos distribuidos en asignaturas. Cuando se entendió que el que aprende construye la comprensión conceptual al disponer de oportunidades para utilizar el conocimiento factual en situaciones concretas, los currículos integrados y los basados en casos fueron siendo adoptados por un número creciente de facultades de medicina. Es a través del aprendizaje experiencial y la observación guiada, participando en la cotidianidad de los escenarios clínicos, que se desarrolla tanto la comprensión conceptual como el reconocimiento de patrones y los procesos de razonamiento clínico para la resolución de problemas [12].

En la atención a los pacientes participan cada vez un mayor número de agentes: diferentes médicos especialistas junto a miembros de otras profesiones sanitarias. Todos ellos contribuyen con sus distintos conocimientos y habilidades, de forma que en un escenario clínico existe un conjunto de saberes a disposición del que aprende que ningún individuo por sí solo puede poseer, lo que ofrece innumerables posibilidades de aprendizaje. Es necesario reconocer que el conocimiento está distribuido y que la atención a los pacientes es una actividad social, cultural e históricamente definida. Los escenarios clínicos en los que se produce la atención a los pacientes, con la inmensa cantidad de conocimientos distribuidos entre los participantes, deben ser tenidos en cuenta por los educadores no tan sólo como lugares idóneos para la adquisición del conocimiento formal explicito básico para la profesión, sino también como un lugar idóneo para transferir el conocimiento tácito y los valores profesionales. Es en estos escenarios clínicos en donde los docentes y tutores exhiben su función de rol-models [8], desplegándose ahí la mayor parte del extenso currículo oculto que cada facultad inconscientemente, pero con gran eficacia, ofrece al estudiante. 
El experto es un individuo cuya actuación en su dominio es excepcional y que además está comprometido con la mejora permanente de su desempeño. La actuación habilidosa se caracteriza por la facilidad en realizar tareas rutinarias y su efectividad en resolver situaciones excepcionales. Para los médicos, la pericia requiere ser eficiente en competencias nucleares que permitan respuestas efectivas en situaciones complejas, así como la capacidad para profundizar y expandirlas innovando [13]. La 'experteza' tiene, pues, una dimensión adaptativa con estrategias para el aprendizaje continuo y la innovación, que es particularmente relevante tanto para los médicos individualmente como para las comunidades de profesionales clínicos.

Uno de los objetivos de la educación médica es asegurar que los estudiantes y los residentes estén preparados para el aprendizaje futuro, con el fin de desarrollar soluciones a problemas más difíciles, complejos o nuevos y desplegar nuevas rutinas para solucionarlos. Si sólo logra producir profesionales habilidosos pero incapaces de afrontar nuevos problemas o aproximaciones novedosas a viejos problemas, expertos en rutinas pero incapaces de crear conocimientos, es decir, faltos de una actitud inquisitiva y de mejorar, el sistema habrá fracasado, pues su objetivo es conseguir 'expertos adaptables' capaces de afrontar nuevas situaciones, reformular soluciones y desarrollar nuevas formas para obtener mejores resultados para sus pacientes [14].

¿Cómo facilitar el desarrollo de la pericia adaptativa? ¿Cómo desarrollar la flexibilidad de las respuestas a la variación de las situaciones, a la modificación de las prácticas establecidas, a atravesar fronteras entre dominios o a explorar nuevas perspectivas? [13]. En primer lugar, se debe fomentar una orientación constructivista de la adquisición del conocimiento, del aprender. Ello supone, de entrada, el fomento de actitudes que favorezcan la capacidad para reconocer que las interpretaciones que se realizan son siempre provisionales y no definitivas, sujetas a cambios $y$ mejoras: entender que las preguntas que se formulan no deben ser cerradas, presuponiendo ya una respuesta determinada, y, finalmente, no presuponer tampoco que ya se posee el conocimiento suficiente intentando completar la comprensión de las situaciones, dando por cierta la mejor hipótesis posible, como se hace con frecuencia.

La adquisición de rutinas libera espacios cognitivos gracias a los automatismos y al recono- cimiento de clichés (pattern recognition). Estos recursos liberados pueden utilizarse para identificar nuevos problemas, soluciones o ampliar el conocimiento [15].

¿Cómo aprender a detectar, seleccionar y enfocar adecuadamente las oportunidades de mejora que ofrece la cotidianidad del quehacer profesional? Schön [16] identifica tres componentes para la formación en la 'reflexión-en-la-acción'. El primero es aprender haciendo tareas asequibles en entornos controlados, simulados y supervisados. El segundo es el coaching, consistente en el demostrar, criticar, aconsejar y cuestionar por parte de los supervisores, con el fin de que los alumnos se reformulen los problemas o las estrategias y adquieran con ello nuevos conocimientos. El tercero es el diálogo reflexivo que ayuda a encontrar alternativas, aprovechando las situaciones inciertas, desconocidas o conflictivas para ejercitarse en la búsqueda de sentido y comprensión, generadoras del aprender permanente sobre la acción.

¿Cómo saber cuándo es posible mejorar la actuación y cómo aprender a desarrollar las estrategias de mejora? Una actitud inquisitiva se acompaña de la capacidad de percibir la insuficiente comprensión de un fenómeno. Una mente inquisitiva se pregunta siempre el porqué de las cosas. La autoevaluación y el aprendizaje autodirigido son aproximaciones útiles pero pueden constituir una insuficiente motivación para generar una actitud inquisitiva. Puede ser necesario aprender a utilizar fuentes externas de información, benchmarks y feedback sobre el desempeño [17]. Aunque gran parte del aprendizaje consiste en adquirir la capacidad de realizar rutinas consistentemente, no se debería olvidar la necesidad de aprender a reconocer las situaciones que demandan soluciones no rutinarias y enfoques novedosos.

El proceso educativo es un proceso transformativo para llegar a devenir miembro funcional de la comunidad médica. Para ello se requiere la adquisición de un vasto cuerpo de conocimientos y habilidades personales que incluyen dimensiones de orden público y social. Estas últimas, según el modelo sociocultural del aprendizaje, se aprenden con el hacer en contextos auténticos, actividades del mundo real, en las que se muestra la verdadera naturaleza social de un quehacer médico que raramente se lleva a cabo en solitario. Aprender en equipo representa progresar desde posiciones marginales hacia posiciones centrales [18]. En el trabajo en equipo, el feedback y el rol-modeling 
constituyen elementos educativos esenciales para desarrollar la capacidad de actuar competentemente. Pero la sociedad demanda más de la medicina que una actuación competente de los médicos. Demanda el compromiso con la mejora, con la excelencia, para lo cual la formación, entendida como un proceso de aculturalización [19], debe atender también al desarrollo moral y emocional [20].

El aprendizaje experiencial en equipos clínicos contribuye a construir la identidad profesional [21] y a adquirir confianza y motivación para aprender y mejorar su competencia [22]. Participando en las comunidades de práctica clínica, los estudiantes y los residentes viven los valores y compromisos del equipo, formando parte de los recursos sociales e intelectuales del grupo, desempeñando el papel que les corresponde e interrelacionándose con los demás miembros. Del grupo obtendrán los 'modelos' que emular, y a medida que vayan progresando en su desarrollo, irán convirtiéndose en modelos para las siguientes cohortes de estudiantes o residentes.

Los aprendices de la profesión tienden a internalizar los valores de la comunidad de práctica, pero cuando en estos contextos las prácticas no se corresponden con los valores que se profesan, el efecto deletéreo de éstas en los noveles aprendices puede ser nefasto. Hafferty [23] describe como 'currículo oculto' el impacto que causa en los que aprenden el hecho de ver cómo se están haciendo las cosas, especialmente si no coinciden con la prédica del cómo deberían hacerse. Entonces, habrá que cuidar los comportamientos docentes en los entornos educativos para no echar a perder el entusiasmo de los que se inician en el aprendizaje de la profesión, pues educación médica es, sobre todo, el proceso de la transformación de identidades.

\section{Puntos clave}

Si la finalidad de la educación médica es mejorar la atención a la salud y el profesionalismo constituye un compromiso con la excelencia en la atención de los pacientes mediante la innovación, así como con la participación en la comunidad profesional, ¿cómo debería diseñarse y llevarse a cabo el proceso educativo de los médicos? Cooke et al [24] proponen la necesidad de cambos en el currículo, la pedagogía y la evaluación, teniendo en cuenta las tres premisas siguientes:
- Aprender es un desarrollo progresivo.

- Aprender es un proceso participativo.

- El aprendizaje es contextualizado y distributivo.

El conocimiento es dinámico porque constantemente se modifica, se recombina, se expande y se elabora creando nuevas comprensiones o mejorando el desempeño. Al novato que realiza paso a paso los procedimientos, cautelosa y meticulosamente, se le escaparán hallazgos que un experto no pasará por alto, mientras que un estudiante más avanzado, además de hacerlo con mayor soltura, puede encontrar relaciones en lo que está haciendo con otras situaciones y así 'aprender haciendo', que es más que aprender a hacerlo. Aprender es, pues, un desarrollo de las capacidades personales que se produce de forma progresiva. Es un desarrollo personal que puede ser guiado y favorecido, o no, por los recursos y la organización docente, pero que se produce en los sujetos movidos por su motivación y gracias a su esfuerzo y perseverancia. Lo que un sujeto sabe y sabe hacer depende de su conocimiento y comprensión, y éstos, de sus experiencias previas y de que las situaciones favorezcan o no el hecho de acceder a nuevo conocimiento y comprensión.

El desarrollo eficiente de un estudiante neófito hasta alcanzar el estatus de médico competente precisa de un currículo diseñado pensando en facilitar esta transformación progresiva, por lo que debe tener una característica básica: dar respuesta a las necesidades del que aprende ofreciendo oportunidades de aprender en entornos de trabajo clínico, de forma supervisada y con la flexibilidad suficiente como para poder permanecer en él el tiempo necesario, y solamente el necesario, con el fin de que el que aprende alcance el objetivo deseado.

Para ello es necesario utilizar las pedagogías acordes con las necesidades de aprendizaje del que aprende y no las convenientes para el que enseña. Estas pedagogías deben permitir al que aprende a participar en la dirección de su aprendizaje y a reconocer cuándo necesita ayuda, para qué y dónde puede encontrarla. Pedagogías que estimulen la curiosidad y creen en el que aprende interés por el aprendizaje para toda la vida.

El sistema de evaluación debe marcar el progreso del aprendizaje en todos los dominios y no tan sólo del conocimiento formal y de las habilidades psicomotoras adquiridas, incorporando facetas actualmente ignoradas. Tan o más importante que valorar el conocimiento formal adqui- 
rido debería ser la evaluación de las respuestas al reconocimiento de lo que no saben, la motivación para aprender y sus estrategias de aprendizaje. Cuestiones que previamente, y al margen de ser evaluadas, deberían considerarse como valiosos objetivos educativos.

La internalización de los valores, la transformación en una nueva identidad y la actuación competente no se logran atendiendo en el aula a las explicaciones eruditas de un experto, o al menos no se logra solamente con dicha pedagogía. Es preciso participar en el trabajo de una comunidad práctica para lograrlo. Trabajando en equipos en los que otros están más avanzados y siendo guiados y supervisados en la realización de las tareas encomendadas es como se aprende a comunicar con los colegas y actuar con sentido ético y consideración cultural.

La estructura curricular debe secuenciar adecuadamente las tareas y actividades para que el que aprende pueda contribuir progresivamente al trabajo del equipo con los pacientes, utilizando pedagogías que involucren a los que aprenden en la comunidad de práctica, con supervisión y guía, clarificando roles y responsabilidades, proporcionando feedback sobre la actuación, ejemplarizando rol-models y evaluando, además del conocimiento y las habilidades, el razonamiento y juicio clínico, la comunicación y la capacidad de aprender y mejorar.

La estructura curricular debe ofrecer experiencias repetidas en múltiples entornos clínicos y centrarse más en cómo se enfocan los problemas. Debe centrarse más en cómo se utiliza la información que en la memorización de datos, además de hacer visible el conocimiento oculto del que disponen los que aprenden, Las pedagogías emplearán la observación y reflexión guiadas, fomentarán las actitudes inquisitivas, el deseo de descubrir y el debate entre pares para que salgan a la luz su comprensión y sus asunciones. La evaluación tendrá en cuenta elementos como el uso que se hace de los recursos y las redes sociales, la actuación colectiva de los equipos y la contribución individual al desempeño colectivo, una evaluación que reconozca que la inteligencia clínica es situacional y distribuida entre todos los miembros del equipo.

Este planteamiento, naturalmente complejo pero al mismo tiempo absolutamente imprescindible para una mejora sostenida y eficaz de la actividad formativa, constituye el hito central de la evolución de los currículos hacia el nuevo modelo de sistema educativo que pretende el proceso de Bolonia. La capacidad de las universidades y los hospitales docentes para construir un proceso educativo que tenga presentes estos conceptos dará con el tiempo la medida del éxito de la reforma.

\section{Bibliografía}

1. Wojtczak A. La declaración y el proceso de Bolonia deben revisarse. Educ Med 2010; 13: 67-70.

2. Wear D, Castellani B. The development of professionalism: curriculum matters. Acad Med 2000; 75: 602-11.

3. Boshuizen $\mathrm{H}, \mathrm{Schmidt} \mathrm{H}$. On the role of biomedical knowledge in clinical reasoning by experts, intermediates, and novices. Cognit Sci 1992; 16: 153-84.

4. Bordage G. Elaborated knowledge: a key to successful diagnostic thinking. Acad Med 1994; 69: 883-5.

5. Norman G. Research in clinical reasoning: past history and present trends. Med Educ 2005; 39: 418-27.

6. Hatala R, Brooks L, Norman G. Practice makes perfect: the critical role of mixed practice in the acquisition of ECG interpretation skills. Adv Health Sci Educ 2003; 12: 17-26.

7. Dreyfuss H, Dreyfuss S. Mind over machine: the power of human intuition and expertise in the era of computer. New York: Free Press; 1986.

8. Ericsson K. An expert-performance perspective of research on medical expertise: the study of clinical performance. Med Educ 2007; 41: 1120-30.

9. Eva K. What every teacher needs to know about clinical reasoning. Med Educ 2005; 39: 98-106.

10. Montgomery K. How doctors think: clinical judgment and the practice of medicine. New York: Oxford University Press; 2006.

11. Croskerry P. The importance of cognitive errors in diagnosis and strategies to minimize them. Acad Med 2003; 78: 775-80.

12. Billet $\mathrm{S}$. Constituting the workplace curriculum. Journal of Curriculum Studies 2006; 38: 31-48.

13. Hatano G, Oura Y. Commentary: reconceptualizing school learning using insight from expertise research. Educ Res 2003; 32: 26-9.

14. Mylopoulos M, Regher G. Cognitive metaphors of expertise and knowledge: prospects and limitations for medical education. Med Educ 2007; 41: 1159-65.

15. Regher G, Mylopoulos M. Maintaining competence in the field: learning about practice, through practice, in practice. J Contin Educ Health Prof 2008; 28: S19-23. 
16. Schön D. Educating the reflexive practitioner. San Francisco: Jossey-Bass; 1987.

17. Eva K, Regher G. Self-assessment in the health professions: a reformulation and research agenda. Acad Med 2005; 80: S46-54.

18. Billet S. Learning in the workplace: strategies for effective practice. Crow Nest, Australia: Allen, Urbin; 2001.

19. Hafferty F, Franks R. The hidden curriculum, ethics teaching, and the structure of medical education. Acad Med 1994; 69: 861-71.

20. Epstein R. Mindful practice. J Am Med Assoc 1999; 282: 833-9.
21. Forsythe G. Identity development in professional education. Acad Med 2005; 80: S112-7.

22. Dornan T, Boshuizen H, King N, Sherpbier A. Experience-based learning: a model linking the process and outcomes of medical students' workplace learning. Med Educ 2007; 41: 84-91.

23. Hafferty F. Beyond curriculum reform: confronting medicine's hidden curriculum. Acad Med 1998; 73: 403-7.

24. Cooke M, Irby DM, O'Brien BC. Educating physicians: a call for reform of medical schools and residency. The Carnegie Foundation for the Advancement of Teaching. San Francisco: Jossey-Bass; 2010. 